\title{
Public Disclosure on Social Media of Identifiable Patient Information by Health Professionals: Content Analysis of Twitter Data
}

Wasim Ahmed ${ }^{1}$, BA, MSc, PhD; Reshma Jagsi ${ }^{2}$, DPhil, MD; Thomas G Gutheil ${ }^{3}$, MD; Matthew S Katz ${ }^{4}$, MD

${ }^{1}$ Department of Marketing, Operations and Systems, Newcastle University Business School, Newcastle University, Newcastle upon Tyne, United Kingdom

${ }^{2}$ Department of Radiation Oncology, Center for Bioethics and Social Science in Medicine, University of Michigan, Ann Arbor, MI, United States

${ }^{3}$ Department of Psychiatry, Beth Israel Deaconess Medical Center, Mass. Mental Health Center, Harvard Medical School, Harvard University, Boston, MA, United States

${ }^{4}$ Department of Radiation Medicine, Lowell General Hospital, Lowell, MA, United States

\section{Corresponding Author:}

Wasim Ahmed, BA, MSc, PhD

Department of Marketing, Operations and Systems

Newcastle University Business School

Newcastle University

5 Barrack Rd

Newcastle upon Tyne, NE1 4SE

United Kingdom

Phone: 441912081704

Email: Wasim.Ahmed@Newcastle.ac.uk

\section{Abstract}

Background: Respecting patient privacy and confidentiality is critical for doctor-patient relationships and public trust in medical professionals. The frequency of potentially identifiable disclosures online during periods of active engagement is unknown.

Objective: The objective of this study was to quantify potentially identifiable content shared on social media by physicians and other health care providers using the hashtag \#ShareAStoryInOneTweet.

Methods: We accessed and searched Twitter's API using Symplur software for tweets that included the hashtag \#ShareAStoryInOneTweet. We identified 1206 tweets by doctors, nurses, and other health professionals out of 43,374 tweets shared in May 2018. Tweet content was evaluated in January 2019 to determine the incidence of instances where names or potentially identifiable information about patients were shared; content analysis of tweets in which information about others had been disclosed was performed. The study also evaluated whether participants raised concerns about privacy breaches and estimated the frequency of deleted tweets. The study used dual, blinded coding for a $10 \%$ sample to estimate intercoder reliability using Cohen $\kappa$ statistic for identifying the potential identifiability of tweet content.

Results: Health care professionals $(\mathrm{n}=656)$ disclosing information about others included 486 doctors $(74.1 \%)$ and 98 nurses (14.9\%). Health care professionals sharing stories about patient care disclosed the time frame in 95 tweets $(95 / 754,12.6 \%)$ and included patient names in 15 tweets $(15 / 754,2.0 \%)$. It is estimated that friends or families could likely identify the clinical scenario described in 242 of the 754 tweets (32.1\%). Among 348 tweets about potentially living patients, it was estimated that 162 (46.6\%) were likely identifiable by patients. Intercoder reliability in rating the potential identifiability demonstrated $86.8 \%$ agreement, with a Cohen $\kappa$ of 0.8 suggesting substantial agreement. We also identified 78 out of 754 tweets $(6.5 \%)$ that had been deleted on the website but were still viewable in the analytics software data set.

Conclusions: During periods of active sharing online, nurses, physicians, and other health professionals may sometimes share more information than patients or families might expect. More study is needed to determine whether similar events arise frequently and to understand how to best ensure that patients' rights are adequately respected.

(J Med Internet Res 2020;22(9):e19746) doi: $\underline{10.2196 / 19746}$ 


\section{KEYWORDS}

Social Media; Twitter; Patient Information; Confidentiality; Health Professionals

\section{Introduction}

\section{Background}

Physicians, nurses, and other health professionals remain among the most trusted professionals in the United States because of their commitment to the well-being of others; they are a trusted source of health information and guidance [1]. Surveys have demonstrated the high trust in health care professionals of the US public with even higher levels of trust in other countries [1-3]. Still recited by many medical students as they become physicians, the Hippocratic Oath reflects the fundamental importance of patient privacy as a critical element of the doctor-patient relationship and a precondition for the trust of the public. In the United States, the Health Insurance Portability and Accountability Act (HIPAA) requires deidentification of data to avoid sharing protected health information [4]. The US Patient's Bill of Rights also states that patients have the right to be able to talk privately with medical professionals and that personal information be protected. Thus, both ethical and legal reasons to maintain patient privacy exist.

Fulfilling physicians' obligations to protect the well-being and privacy of their patients is complicated in the age of the internet. Internet culture is very different from that of the medical profession, creating potential ethical problems with boundaries and privacy that did not exist when physicians interacted exclusively offline. In order to maintain the trust of the public and that of individual patients, physicians increasingly need to understand the limits and risks of disclosure of certain types of information online. Although concerns about unprofessional medical student and resident behavior online have been articulated before [5,6], the ethical risks of public disclosure, when narrative medicine intersects with social media, remain poorly defined $[7,8]$.

Social media usage has become popular among medical professionals. A survey in 2014 by QuantiaMD [9] found that, of 4000 physicians surveyed, $90 \%$ noted that they used some form of social media for personal activities, and $65 \%$ used social media for professional reasons. In May 2018, thousands of individuals - including many health care professionals — shared health-related stories on Twitter using the hashtag \#ShareAStoryInOneTweet in response to one physician's spontaneous tweet of a patient story that included this hashtag. Certain tweets included potentially identifiable information that could be considered a breach of confidence when disclosed without patient consent, risking harm to patients, physician's careers, and public trust in the profession.

An article in July 2018 [10] highlighted the importance of sharing stories but did not address the potential risks of sharing online. Over time, some viral tweets have been deleted, raising further concerns that the platform allowing easy disclosure might have led to posts that authors subsequently regretted. A notable example of a popular post (altered to avoid identification) was retweeted 13,491 times and liked by 55,994 people before being deleted:
I delivered a baby very underweight, weighing two pounds. They said he did not have a chance. I remained with him for a couple of days. Nine years later, he played his first football game last week.

Hashtags can make online content searchable and discoverable online, regardless of time since publication [11]. The American Medical Association, Massachusetts Medical Society, and other organizations advise physicians to report unprofessional social media use [12,13]. What constitutes unprofessional behavior on social media is not clearly defined. To advance a common understanding and to facilitate subsequent discussion within the profession about what is appropriate, we sought to describe participation of physicians and other health professionals in this event, the reach of their postings, and the occurrence of potentially identifiable disclosures about patients.

\section{Related Work}

Early research on health professionals using Weblogs [14] examined 271 medical blogs, finding that individual patients were described in 114 blogs, and 45 blogs had enough information for patients to identify themselves. Scholars have questioned whether it would ever be ethical for medical professionals to write publicly about patients without their consent [15]. Previous work [16] where young doctors on Facebook were studied has also specifically highlighted privacy issues by finding that some of the private information shared by the doctors (the doctors' own private information shared by the doctors themselves) could bring the profession into disrepute. Previous work [17] has also noted that the use of social networking sites such as Twitter and Facebook by doctors can lead to complaints by patients.

Despite the importance of previous work [16,17], there appears to be a lack of empirical research on the use of popular hashtags for sharing patient stories by medical professionals. Understanding information sharing using hashtags, such as \#ShareAStoryInOneTweet, is important because social media is becoming more ingrained in society, and potential privacy violations may exist in this context. Furthermore, as social media use increases, online disclosure of private information via social media is likely to remain an issue for health care systems around the world. However, recent research [18] has also highlighted the positive role medical professionals could play on social media, for instance, by countering medical misinformation.

The results of this study are likely to be of interest to those compiling guidelines for the use of social media by medical professionals.

\section{Research Questions and Objectives}

The overall research aim of this study was to develop a better understanding of the content shared with the hashtag \#ShareAStoryInOneTweet.

The objectives of the study were to (1) identify unique tweets sent by doctors and other health care providers using the hashtag, (2) to develop an understanding of the characteristics of the 
doctors and health care providers using the hashtag, and (3) to categorize tweets into themes and identify the frequency of instances in which patients could be identified by themselves or by their family.

\section{Methods}

\section{Cohort Definition}

Because all information about the published content was publicly accessible, Lowell General Hospital approved this study as institutional review board exempt. To evaluate content in the \#ShareAStoryInOneTweet phenomenon, Symplur Signals (Symplur LLC), a proprietary health care-focused database and analytics program collecting data on Twitter using its Enterprise application program interface, was utilized [19]. The first tweet with the hashtag occurred May 4, 2018. From May 4, 2018 through December 31, 2018, 45,040 tweets that included the hashtag \#ShareAStoryInOneTweet were identified. The study focused on 43,374 tweets shared in the month of May 2018 (midnight May 1 to midnight June 1, Eastern Standard Time). The analysis was conducted in January 2019. Using the software program, we identified tweets from doctors, patients, and other health care stakeholders (eg, caregivers, pharmaceutical firms, academic, or research organizations), based upon public self-identification in their Twitter profiles (ie, by identifying information provided within their Twitter biography) [20]. There were 4871 tweets identified, of which, 1206 were unique (the remainder represented retweeting of prior postings).
Unique tweets were reviewed by reading text provided within the data set and then evaluating the URL and each account's public profile on Twitter's website as of March 2019. Tweets from students misclassified as health care providers (1.3\%) (eg, those listing "future doctor" in profile), from blocked accounts $(0.3 \%)$, or with no relevant content posted with the hashtag (1.2\%) were excluded, leaving a total of 1172 tweets shared by physicians, nurses, or other health professionals (Figure 1). The study also excluded 26 retweets in which the authors used the hashtag to share someone else's disclosure rather than their own, and 127 that included the author's own illness experiences rather than those of others. The study also excluded 78 tweets $(6.5 \%)$ with content found in the data set but deleted from Twitter when evaluated on the website.

Characteristics of the health professionals sharing these tweets was examined, using information publicly available in their online profiles, including profession, gender, and country. Physicians were also categorized by specialty as described in their profiles or as unknown if not stated. More detailed content analysis focused upon the tweets in which the health care professional shared the illness or clinical experience of someone other than themselves.

The study also evaluated tweets commenting upon the hashtag-related phenomenon or recommending participation to others. The study analyzed tweets individually rather than as content threads. 
Figure 1. Tweets by doctors and other health care providers during \#ShareAStoryInOneTweet in May 2018. HCP: health care providers.

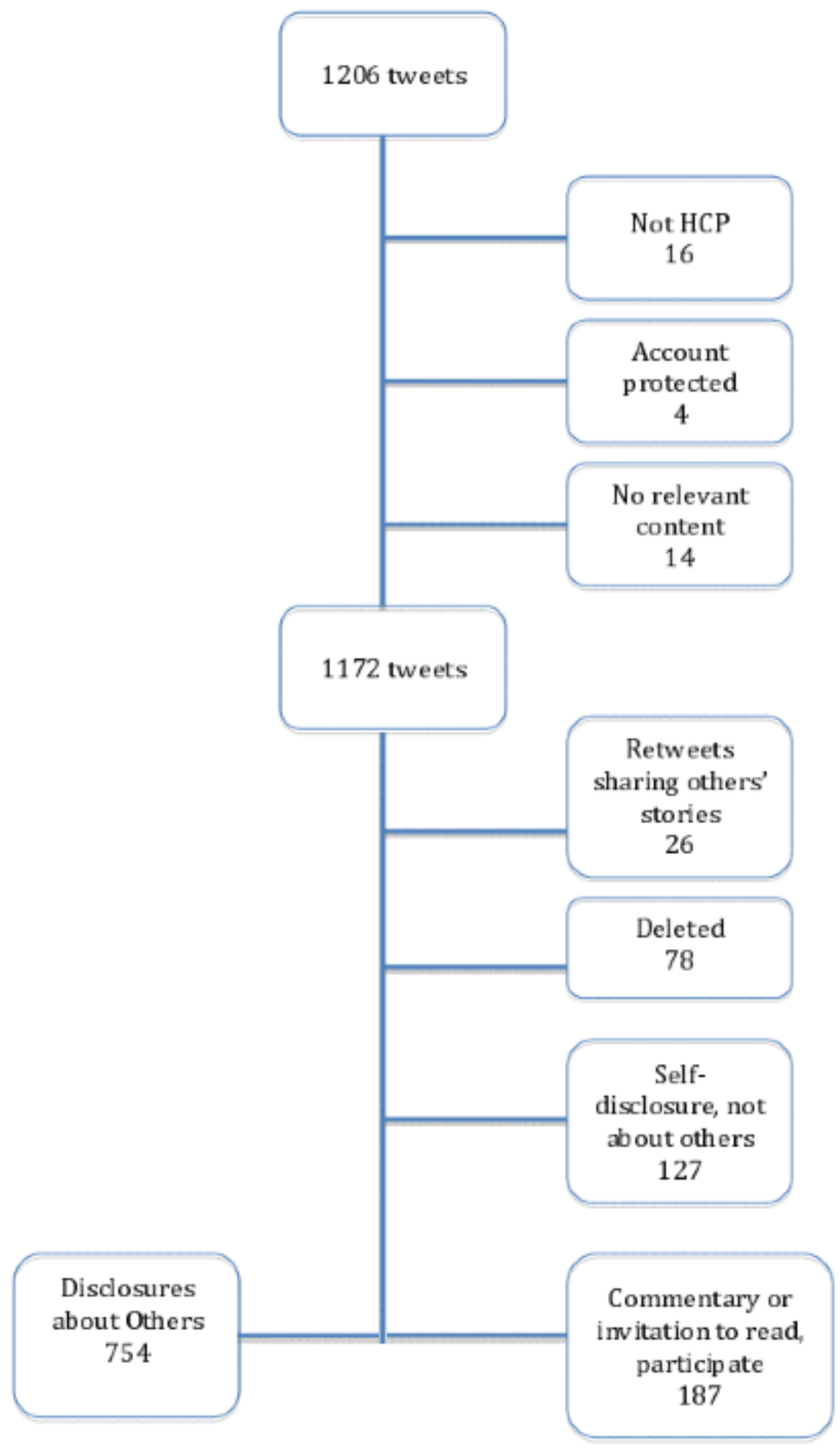

\section{Measures}

To assess the magnitude of hashtag use, the study evaluated total number of tweets. We also evaluated the number of total participants, focusing upon physician, nurse, and other health care professionals. We calculated tweets per hour, number of tweets, and use of images both in aggregate and by health care stakeholder categories. In order to capture hourly tweet activity rates, we restricted the time frame to the first two weeks starting

May 4th to focus on the viral period. We evaluated the potential reach of the tweets using the software's definition of impressions-follower count at the time of each tweet's publication online (eg, a doctor posting while having 500 followers was equal to 500 impressions).

For each tweet, we coded several measures: the tweet author's role in the other person's clinical care; whether the patient died or was dying; whether the author helped save the other person's life; inclusion of patient name; inclusion of a clinical image; 
and inclusion of a specific age. We categorized the time frame of the event described within a tweet as within the past year, 1-2 years ago, 2-5 years ago, >5 years, or unknown.

Whether either a patient or the patient's family or friends would be able to identify the clinical scenario described in each tweet was categorized broadly in response to codebook question "Could patient or family potentially identify the clinical situation?" as yes (more likely than not to be identifiable) or no (not likely to be identifiable). If it was unclear, the code indeterminate was applied and was considered in analyses to be no.

One author (MK) assessed all tweets; a second author (WA) coded a $10 \%$ sample independently. Intercoder reliability and percentage agreement were assessed using ReCal [21]. The two authors then reached consensus on discrepancies and used this exercise to identify any areas where the first coder might systematically have erred.

Because the tweets could be discoverable in malpractice or tort suits, we also analyzed whether the author made comments with a negative opinion about the patient or family, or if the author acknowledged that a medical error occurred. We also assessed whether information was shared about vulnerable patients, as defined by the US Department of Health and Human Services [22].

We separately evaluated tweets commenting upon the hashtag to determine whether the authors had a favorable or unfavorable opinion of the viral sharing, or if they invited others to share stories or to participate. We also identified whether these tweets expressed any concern about privacy breaches.

\section{Statistical Analysis}

Overall activity and frequencies for stakeholder participation using Symplur. Frequencies, median, and mean endpoints for content analysis were calculated using Excel (for Mac 2011 version 14.7.2, Microsoft Inc). Cohen $\kappa$ was used to measure interrater reliability [23].

\section{Results}

\section{Tweet Volume}

For May 2018, we identified 31,690 individuals who posted tweets with the hashtag, with a potential of 106.5 million views; $1725(5.3 \%)$ individuals self-identifying as doctors and 861 $(2.6 \%)$ as other health care providers shared tweets. At its peak, activity showed 1274 tweets per hour (Figure 2).

Figure 2. Tweets per hour including the hashtag \#ShareAStoryInOneTweet in May 2018.

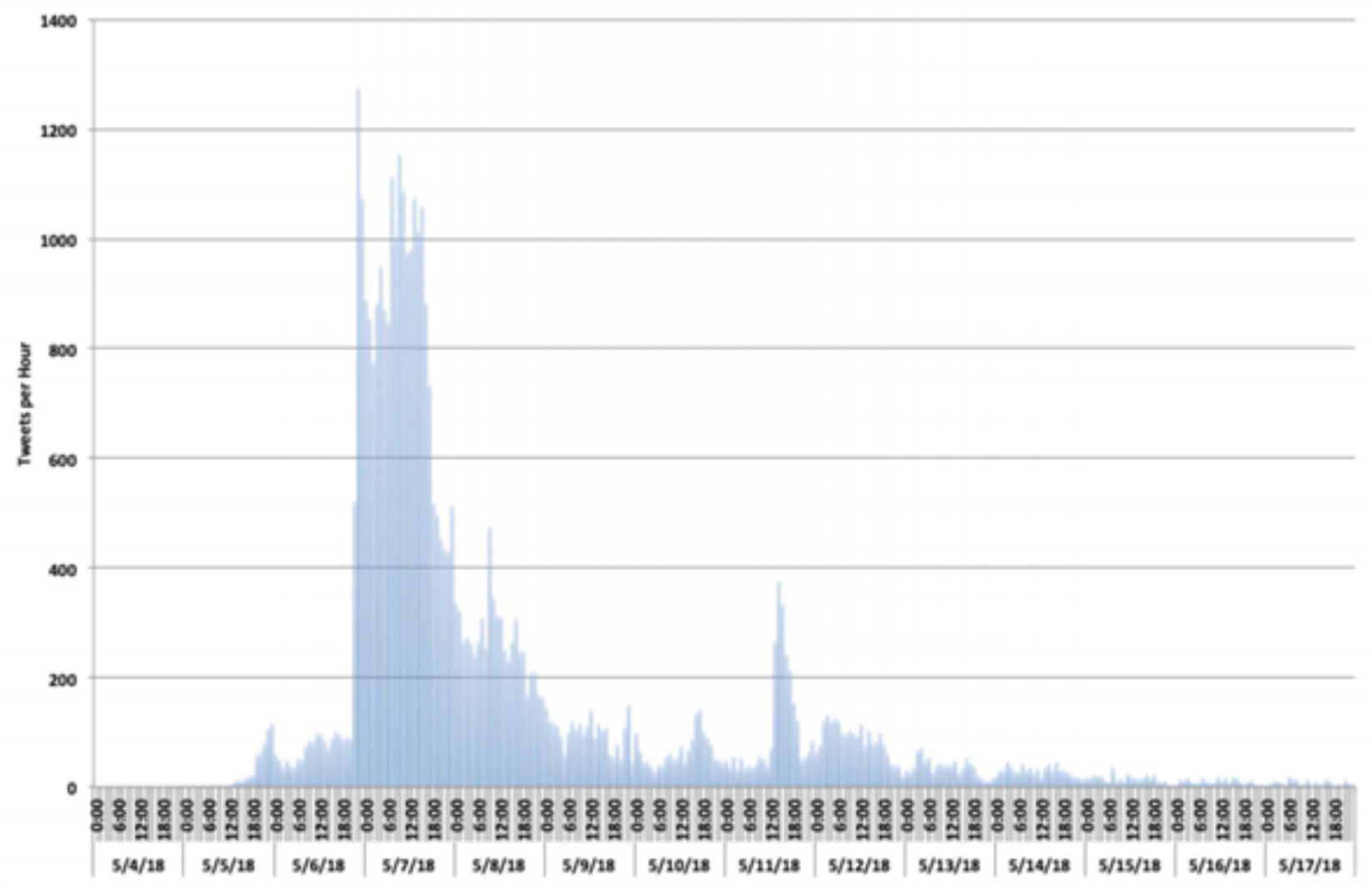

\section{Tweets With Disclosures About Others}

The characteristics of the health professionals sharing tweets with disclosures about others are presented in Table 1. Of the 656 health professionals, 384 (58.5\%) were female; physicians - emergency medicine, family medicine or general practice, and hematology-oncology were the specialties most frequently represented-constituted the largest proportion of the tweeters $(486 / 656,74.1 \%)$, with nurses representing a minority $(98 / 656,14.9 \%)$; and most were in the United States $(347 / 656,52.9 \%)$, followed by Canada $(99 / 656,15.1 \%)$ and the United Kingdom (82/656, 12.5\%). 
The majority $(659 / 754,87.4 \%)$ involved the sharing of stories about direct patient care, rather than the author's role as a family caregiver or in another role (Table 2), and 13.6\% (95/754) of tweets included a specific time frame. The patient's age was included in 163 of the 754 tweets $(21.6 \%)$, and patient name was included in 15 of the $754(2.0 \%)$. Of the 754,11 tweets (1.5\%) shared a clinical image, 152 tweets (20.2\%) shared information about people in a vulnerable category. Only, 2 tweets $(0.3 \%)$ mentioned patient consent to share within the tweet, one explicit and one inferred from past patient agreement to share a specific story. Based upon the number of likes, a minimum of 154,900 accounts viewed these 754 tweets.

Nearly half of the tweets $(337 / 754,44.7 \%)$ described a clinical scenario involving death or dying. Comments disclosing medical errors $(6 / 754,0.8 \%)$ or expressing a negative opinion about the patient or family were rare $(4 / 754,0.5 \%)$. Agreement between coders was $86.8 \%$, and intercoder reliability Cohen $\kappa=0.8$ suggested substantial agreement [16]. Disagreements in coding occurred mostly between the categories no unclear, which led to the decision to combine the categories for further analysis. We estimated that almost one-third $(242 / 754,32.1 \%)$ of families or friends would likely find the content in the tweet identifiable. Among patients who were potentially still living, the study estimated that nearly half $(162 / 348,46.6 \%)$ contained likely identifiable information, of which $81(50 \%)$ were likely identifiable by families and friends. The 754 tweets received a median of 2 retweets (range: 0-19; total 959) and 16 likes (range: $0-56$; total 735). 
Table 1. Characteristics of doctors and health care professionals sharing tweets disclosing information about others.

\begin{tabular}{|c|c|}
\hline Characteristics & Value $(\mathrm{N}=656), \mathrm{n}(\%)$ \\
\hline \multicolumn{2}{|l|}{ Gender } \\
\hline Female & $384(58.5)$ \\
\hline Male & $266(40.5)$ \\
\hline Unknown & $6(0.9)$ \\
\hline \multicolumn{2}{|l|}{ Profession } \\
\hline Doctor & $486(74.1)$ \\
\hline Anesthesia & $22(4.5)$ \\
\hline Cardiology & $25(5.1)$ \\
\hline Critical care & $12(2.5)$ \\
\hline Emergency medicine & $77(15.8)$ \\
\hline Family medicine-general practitioner & $48(9.9)$ \\
\hline Gastroenterology & $6(1.2)$ \\
\hline Hematology-oncology & $39(8.0)$ \\
\hline Hospitalist & $5(1.0)$ \\
\hline Infectious disease & $5(1.0)$ \\
\hline Internal medicine & $12(2.5)$ \\
\hline Neurosurgery & $5(1.0)$ \\
\hline Obstetrics and gynecology & $13(2.7)$ \\
\hline Palliative care & $18(3.7)$ \\
\hline Pathology & $9(1.9)$ \\
\hline Pediatrics & $28(5.8)$ \\
\hline Psychiatry & $7(1.4)$ \\
\hline Pulmonary medicine & $7(1.4)$ \\
\hline Radiation oncology/clinical oncology & $10(2.1)$ \\
\hline Radiology & $6(1.2)$ \\
\hline Surgery & $19(3.9)$ \\
\hline Trauma surgery & $20(4.1)$ \\
\hline Unknown & $36(7.4)$ \\
\hline Other & $57(11.7)$ \\
\hline Nurse & $98(14.9)$ \\
\hline Nurse, not otherwise specified & $66(67.3)$ \\
\hline Critical care & $16(16.3)$ \\
\hline Emergency medicine & $8(8.2)$ \\
\hline Other & $8(8.2)$ \\
\hline Nurse practitioner & $12(1.8)$ \\
\hline Paramedic & $18(2.7)$ \\
\hline Pharmacist & $7(1.1)$ \\
\hline Physical therapy & $10(1.5)$ \\
\hline Psychologist & $5(0.8)$ \\
\hline Social worker & $7(1.1)$ \\
\hline Speech therapy & $5(0.8)$ \\
\hline Other & $8(1.2)$ \\
\hline
\end{tabular}




\begin{tabular}{ll}
\hline Characteristics & Value $(\mathrm{N}=656), \mathrm{n}(\%)$ \\
\hline Country & $11(1.7)$ \\
Australia & $99(15.1)$ \\
Canada & $7(1.1)$ \\
India & $16(2.4)$ \\
Ireland & $7(1.1)$ \\
Saudi Arabia & $4(0.6)$ \\
South Africa & $4(0.6)$ \\
Spain & $82(12.5)$ \\
United Kingdom & $347(52.9)$ \\
United States & $55(8.3)$ \\
Unknown & $24(3.7)$ \\
Other & \\
\hline
\end{tabular}

Table 2. Content characteristics tweets with disclosures about others.

\begin{tabular}{|c|c|}
\hline Content characteristic & Value $(\mathrm{N}=754), \mathrm{n}(\%)$ \\
\hline \multicolumn{2}{|l|}{ Author role } \\
\hline Health care professional & $669(88.7)$ \\
\hline Patient & $0(0.0)$ \\
\hline Caregiver & $42(5.6)$ \\
\hline Other & $43(5.7)$ \\
\hline \multicolumn{2}{|l|}{ Time frame described } \\
\hline Within past year & $5(0.7)$ \\
\hline 1-2 years & $6(0.8)$ \\
\hline $2-5$ years & $5(0.7)$ \\
\hline$>5$ years & $79(10.5)$ \\
\hline Unknown/not described & $659(87.4)$ \\
\hline \multicolumn{2}{|l|}{ Content } \\
\hline Author involved in patient care & $635(84.2)$ \\
\hline Dying patient or patient death & $337(44.7)$ \\
\hline Saving a patient's life & $131(17.4)$ \\
\hline Include a clinical image & $11(1.5)$ \\
\hline Include patient name & $15(2.0)$ \\
\hline Provide specific patient age & $163(21.6)$ \\
\hline Express negative opinion of patient or family & $4(0.5)$ \\
\hline Mention medical error & $6(0.8)$ \\
\hline \multicolumn{2}{|l|}{ Estimated likely } \\
\hline Can family or friends identify situation described? & $242(32.1)$ \\
\hline \multicolumn{2}{|l|}{ Can patient identify situation described? } \\
\hline All tweets & $183(24.3)$ \\
\hline Potentially living patients $(n=348)$ & $162(46.6)$ \\
\hline Vulnerable population & $152(20.2)$ \\
\hline
\end{tabular}




\section{Tweets Relating to the Hashtag}

Of 187 tweets actively part of the conversations without disclosures, 173 made some commentary: 6 tweets (3.2\%) raised concerns about privacy or identifiable information in the tweets with disclosures, $1(0.6 \%)$ tweet involved another critical comment, and 167 (96.5\%) tweets were neutral or favorable. Of 187 tweets, 42 tweets $(22.5 \%)$ invited others to read the hashtag's stream or contribute to it.

\section{Discussion}

\section{Principal Findings}

This retrospective study describes a physician-initiated event sharing health-related stories and information on Twitter by quantifying the global participation of health care professionals and the type of content shared. The tweeted stories became widely shared, attracting media attention and disseminating the information widely. Almost none (either explicitly or appear to) confirm consent to share information publicly on the popular social network. Nurses, physicians, and other health professionals commenting using the hashtag were more likely to express support for the event and encourage others to participate than they were to raise concerns about patient privacy breaches. However, recent research suggests that $12 \%$ of patients may have less trust in physicians describing patient stories on social media, even if shared respectfully [24].

The study showed a relatively high incidence of sharing stories including details that might make them potentially identifiable to patients themselves or to families and friends in a setting that involved a large number of health care professionals. This finding highlights a lack of awareness about the privacy issues intrinsically connected to interactions on social media. Early in the use of social media, most US state medical boards received at least one report of an episode of online professionalism violations for disciplinary action, including violations of patient confidentiality [25]. Although surveys of medical students and physicians suggest the incidence of unprofessional behavior among medical students is infrequent [26,27], this study indicates that in some circumstances health care professionals may share more information publicly than the public might expect. Privacy breaches risk potential negative effects on physician-patient relationships, professional disciplinary actions or torts, and eroding public trust.

The findings of this study differ from those of prior studies $[16,17]$ of online medical professionalism at least partly because we analyzed, in detail, one specific event focused upon health-related disclosures. There is no indication this episode was planned, and the incidence of similar episodes is unknown. However, it was not an isolated event; for example, another prominent example involved physicians opposing gun violence, who used the hashtag \#ThisIsOurLane on Twitter in November 2018 [28]. Physicians focused on policy issues, but some may have failed to recognize privacy concerns, publishing tweets with photographs similar in nature to prior social media content in other cases, resulted in professional termination $[29,30]$. Social media studies publishing tweets often permit reverse identification of the authors [31], and a survey suggests that most participants are somewhat or very uncomfortable with their tweets being quoted in a published research paper [32]. It was found that $6.5 \%$ of tweets archived in the software's archived data set were in fact deleted by health care professionals, indicating that some did not want their tweets to remain publicly visible. Even if deleted online, tweets may retain permanence and discoverability, when published in journals.

Most research evaluating online disclosures focus on the privacy paradox, in which people value their privacy but still share their own information. Surveys indicate people may value short-term social rewards of self-disclosure online more than long-term privacy concerns [33], and high social capital of social network users is associated with increased self-disclosures over time [34]. For people disclosing information about others, the research is more limited, but opinion leadership and female gender have been linked to less concern about others' privacy [35], consistent with the findings of this study. Health care professionals may be prone to these same tendencies, despite their training and education to maintain privacy. Generational differences in concerns about privacy online may also play a role [36], but assessment of this possibility was not within the scope of this study.

Based upon the temporal pattern of sharing, this hashtag-related event may be less similar to narrative medicine and writing and more similar to a brief episode of social contagion, in which viral sharing of content or emotions online may occur and involve more than simple, conscious risk-reward tradeoffs [37,38]. Unlike traditional peer-reviewed publication of a medical story in narrative medicine, tweeting occurs quickly and does not permit editing. The observation that $6.5 \%$ chose later to delete their contributions may suggest that some health care professionals who participated in the experience may have later viewed their behavior as a temporary lapse in judgment.

Another contributing factor may be a knowledge gap for physicians and other health care professionals on how to behave online. While many recognize the importance of online professionalism, curricula for use in formal medical education are only beginning to emerge and remain uncommon [39-41]. Of note, the ethical obligation to maintain confidentiality does not end with a patient's death [42]. The digital medium does not avoid the potential that disclosures about patients risk breaching confidentiality, undermining trust within that therapeutic relationship as well as public trust in the medical profession. The findings of this study suggest a potential need for evidence-based training in ethical digital communications skills for undergraduate, graduate, and continuing medical education. Professional societies could create resources that allow social media authors to document having obtained consent, so that disclosing identifiable patient information without consent does not inadvertently become normalized.

\section{Limitations}

This study had several limitations. First, this study examined a very specific event that may occur during very active periods of online engagement but likely overestimated the general incidence of online behaviors that could, in some cases, constitute violations of medical professionalism. Future research could analyze a broader collection of social media posts by 
medical professionals. Second, the study could not assess the number of people actually seeing these tweets; only the number of likes was measured, and the potential reach was estimated. Third, the study only analyzed tweets from accounts that the software identified as health care professionals. The evaluation of all tweets in the cohort confirms the software rarely misclassified nonprofessionals into this group, but the study did not evaluate any other participants in the event to determine if the study could identify more participating health care professionals not categorized as doctors or nurses by the software, which could decrease or increase the incidence of potential privacy breaches. Fourth, by analyzing only tweets with the hashtag, the study potentially underestimated the frequency of others expressing concern about patient privacy. Fifth, given the brevity inherent to the medium of Twitter, it is possible that some authors did indeed have formal documentation of patients' consent to share their stories but that there was insufficient room to include due to character limits in each post. Finally, the assessment of identifiability in this study may differ from those in other studies, and we cannot exclude the possibility that some physicians and nurses tweeting what seemed to be identifiable stories consciously changed important details to deidentify. It was beyond the scope of this study to confirm whether any harm occurred.

Despite these limitations, the findings of this study clearly show that internet-based sharing raises potential pitfalls for medical professionalism. The internet provides nurses, physicians, and other professionals the opportunity to help or harm others on a global scale. Although internet culture may favor maximizing transparency, it can also pose the risk of directly contradicting health professionals' fiduciary duty: first, do no harm, including harm that may be inflicted by what we say.

\section{Conclusion}

The study identified a high incidence of potential privacy breaches online. More research is essential to confirm the findings of this study and determine how to ensure physicians, nurses, and other professionals adapt their behavior to maintain medical professionalism in the digital age. Our results suggest that some who were using the hashtag may not have appreciated that the information being shared might breach patients' privacy We recommend greater specification of professional ethical standards in this context along with evidence-based training in ethical digital communications skills for the undergraduate, graduate, and continuing medical education.

\section{Conflicts of Interest}

WA and TGG have nothing to disclose. MSK reports common stock ownership in Dr. Reddy's Laboratories, Healthcare Services Group, Mazor Robotics, and US Physical Therapy. RJ has stock options as compensation for an advisory board role in Equity Quotient, a company that evaluates culture in health care companies. RJ has received personal fees from Amgen and Vizient and grants for unrelated work from the National Institutes of Health, the Doris Duke Foundation, the Greenwall Foundation, the Komen Foundation, and Blue Cross Blue Shield of Michigan for the Michigan Radiation Oncology Quality Consortium. RJ has a contract to conduct an investigator initiated study with Genentech. RJ has served as an expert witness for Sherinian and Hasso and Dressman Benzinger LaVelle. RJ is an uncompensated founding member of TIME'S UP Healthcare and a member of the Board of Directors of ASCO.

\section{References}

1. Brenan M. Nurses again outpace other professions for honesty, ethics. Gallup. 2018 Dec 20. URL: https://news.gallup.com/ poll/245597/nurses-again-outpace-professions-honesty-ethics.aspx [accessed 2020-08-25]

2. Blendon RJ, Benson JM, Hero JO. Public Trust in Physicians - U.S. Medicine in International Perspective. N Engl J Med 2014 Oct 23;371(17):1570-1572. [doi: 10.1056/nejmp1407373]

3. Huang EC, Pu C, Chou Y, Huang N. Public Trust in Physicians-Health Care Commodification as a Possible Deteriorating Factor: Cross-sectional Analysis of 23 Countries. INQUIRY 2018 Mar 05;55:004695801875917. [doi: 10.1177/0046958018759174]

4. Office for Civil Rights. Guidance regarding methods for de-identification of protected health information in accordance with the Health Insurance Portability and Accountability Act. Health Information Privacy. 2016 Nov 06. URL: https://www. hhs.gov/hipaa/for-professionals/privacy/special-topics/de-identification/index.html [accessed 2020-08-25]

5. Thompson LA, Dawson K, Ferdig R, Black EW, Boyer J, Coutts J, et al. The intersection of online social networking with medical professionalism. J Gen Intern Med 2008 Jul;23(7):954-957 [FREE Full text] [doi: 10.1007/s11606-008-0538-8] [Medline: $\underline{18612723}$ ]

6. Chretien KC. Online Posting of Unprofessional Content by Medical Students. JAMA 2009 Sep 23;302(12):1309. [doi: 10.1001/jama.2009.1387]

7. Ofri D. The Passion and the Peril. Academic Medicine 2015;90(8):1005-1006. [doi: 10.1097/acm.0000000000000672]

8. Wells DM, Lehavot K, Isaac ML. Sounding Off on Social Media. Academic Medicine 2015;90(8):1015-1019. [doi: 10.1097/acm.0000000000000668]

9. Ventola CL. Social media and health care professionals: benefits, risks, and best practices. P T 2014 Jul;39(7):491-520 [FREE Full text] [Medline: 25083128]

10. Rosenbaum L. Twitter Tailwinds — Little Capsules of Gratitude. N Engl J Med 2018 Jul 19;379(3):209-211. [doi: 10.1056/nejmp1806737] 
11. Ahmed W. Public health implications of \#ShoutYourAbortion. Public Health 2018 Oct;163:35-41. [doi: 10.1016/j.puhe.2018.06.010]

12. American Medical Association. Professionalism in the Use of Social Media. American Medical Association. 2016 Nov 14. URL: https://www.ama-assn.org/delivering-care/ethics/professionalism-use-social-media [accessed 2025-08-25]

13. MMS Physicians' Guide to Social Media. Massachusetts Medical Society. 2015. URL: http://www.massmed.org/ Governance-and-Leadership/Committees,-Task-Forces-and-Sections/MMS-Physicians--Guide-to-Social-Media-(pdf)/ [accessed 2020-08-25]

14. Lagu T, Kaufman EJ, Asch DA, Armstrong K. Content of Weblogs Written by Health Professionals. J GEN INTERN MED 2008 Jul 23;23(10):1642-1646. [doi: 10.1007/s11606-008-0726-6]

15. Chretien KC, Kind T. Social Media and Clinical Care. Circulation 2013 Apr 02;127(13):1413-1421. [doi: 10.1161/circulationaha.112.128017]

16. MacDonald J, Sohn S, Ellis P. Privacy, professionalism and Facebook: a dilemma for young doctors. Med Educ 2010 Aug;44(8):805-813. [doi: 10.1111/j.1365-2923.2010.03720.x] [Medline: 20633220]

17. Rimmer A. Doctors' use of Facebook, Twitter, and WhatsApp is the focus of 28 GMC investigations. BMJ 2017 Sep 01:j4099. [doi: 10.1136/bmj.j4099]

18. Ahmed W, Vidal-Alaball J, Downing J, López Seguí F. COVID-19 and the 5G Conspiracy Theory: Social Network Analysis of Twitter Data. J Med Internet Res 2020 May 6;22(5):e19458. [doi: 10.2196/19458]

19. Authentication. Symplur. URL: https://docs.symplur.com/reference\#authentication [accessed 2020-08-25]

20. Utengen A. Health care Stakeholder Segmentation. Symplur. URL: https://help.symplur.com/en/articles/103684-health [accessed 2020-08-25]

21. Freelon D. ReCal: Intercoder reliability calculation as a web service. Int J Internet Science 2010;5(1):20-33. [doi: 10.4135/9781412963947.n228]

22. Vulnerable Populations. Office for Human Research Protections. URL: https://www.hhs.gov/ohrp/regulations-and-policy/ guidance/vulnerable-populations/index.html [accessed 2020-08-25]

23. McHugh ML. Interrater reliability: the kappa statistic. Biochem Med 2012:276-282. [doi: 10.11613/bm.2012.031]

24. Fatollahi JJ, Colbert JA, Agarwal P, Lee JL, Lehmann EY, Yuan N, et al. The Impact of Physician Social Media Behavior on Patient Trust. AJOB Empir Bioeth 2020 Oct 30;11(2):77-82. [doi: 10.1080/23294515.2019.1678533] [Medline: 31663810]

25. Greysen SR, Chretien KC, Kind T, Young A, Gross CP. Physician violations of online professionalism and disciplinary actions: a national survey of state medical boards. JAMA 2012 Mar 21;307(11):1141-1142. [doi: 10.1001/jama.2012.330] [Medline: 22436951]

26. Thompson LA, Black E, Duff WP, Paradise Black N, Saliba H, Dawson K. Protected health information on social networking sites: ethical and legal considerations. J Med Internet Res 2011 Jan 19;13(1):e8 [FREE Full text] [doi: 10.2196/jmir.1590] [Medline: 21247862]

27. Brynolf A, Johansson S, Appelgren E, Lynoe N, Edstedt Bonamy A. Virtual colleagues, virtually colleagues--physicians' use of Twitter: a population-based observational study. BMJ Open 2013;3(7):1-5 [FREE Full text] [doi: 10.1136/bmjopen-2013-002988] [Medline: 23883885]

28. Ranney ML, Betz ME, Dark C. \#ThisIsOurLane — Firearm Safety as Health Care's Highway. N Engl J Med 2019 Jan 31;380(5):405-407. [doi: 10.1056/nejmp1815462]

29. ER Doc forgets patient information is private, gets fired for Facebook overshare. Above the Law. 2011 Apr 29. URL: https:/ /abovethelaw.com/2011/04/er-doc-forgets-patient-info-is-private-gets-fired-for-facebook-overshare/ [accessed 2020-08-25]

30. 'New York Med' nurse fired for 'insensitive' Instagram shot. New York Post. 2014 Aug 14. URL: https://nypost.com/2014/ 07/08/new-york-med-nurse-katie-duke-fired-for-insensitive-instagram-shot/ [accessed 2020-08-25]

31. Ayers JW, Caputi TL, Nebeker C, Dredze M. Don't quote me: reverse identification of research participants in social media studies. NPJ Digit Med 2018 Aug 2;1(1):30 [FREE Full text] [doi: 10.1038/s41746-018-0036-2] [Medline: $\underline{31304312]}$

32. Fiesler C, Proferes N. "Participant" Perceptions of Twitter Research Ethics. Social Media + Society 2018 Mar 10;4(1):205630511876336. [doi: 10.1177/2056305118763366]

33. Hallam C, Zanella G. Online self-disclosure: The privacy paradox explained as a temporally discounted balance between concerns and rewards. Computers in Human Behavior 2017 Mar;68:217-227. [doi: 10.1016/j.chb.2016.11.033]

34. Trepte S, Reinecke L. The reciprocal effects of social network site use and the disposition for self-disclosure: A longitudinal study. Computers in Human Behavior 2013 May;29(3):1102-1112. [doi: 10.1016/j.chb.2012.10.002]

35. Koohikamali M, Peak DA, Prybutok VR. Beyond self-disclosure: Disclosure of information about others in social network sites. Computers in Human Behavior 2017 Apr;69:29-42. [doi: 10.1016/j.chb.2016.12.012]

36. Pereira S, Robinson JO, Peoples HA, Gutierrez AM, Majumder MA, McGuire AL, et al. Do privacy and security regulations need a status update? Perspectives from an intergenerational survey. PLoS One 2017 Sep 19;12(9):e0184525 [FREE Full text] [doi: 10.1371/journal.pone.0184525] [Medline: 28926626]

37. Christakis NA, Fowler JH. Social contagion theory: examining dynamic social networks and human behavior. Statist. Med 2012 Jun 18;32(4):556-577. [doi: 10.1002/sim.5408] 
38. Alshamsi A, Pianesi F, Lepri B, Pentland A, Rahwan I. Beyond Contagion: Reality Mining Reveals Complex Patterns of Social Influence. PLoS One 2015 Aug 27;10(8):e0135740 [FREE Full text] [doi: 10.1371/journal.pone.0135740] [Medline: 26313449]

39. Langenfeld SJ, Vargo DJ, Schenarts PJ. Balancing Privacy and Professionalism: A Survey of General Surgery Program Directors on Social Media and Surgical Education. J Surg Educ 2016 Nov;73(6):e28-e32. [doi: 10.1016/j.jsurg.2016.07.010] [Medline: 27524278]

40. Gomes AW, Butera G, Chretien KC, Kind T. The Development and Impact of a Social Media and Professionalism Course for Medical Students. Teach Learn Med 2017 Mar 08;29(3):296-303. [doi: 10.1080/10401334.2016.1275971] [Medline: 28272900]

41. Flickinger TE, O'Hagan T, Chisolm MS. Developing a Curriculum to Promote Professionalism for Medical Students Using Social Media: Pilot of a Workshop and Blog-Based Intervention. JMIR Medical Education 2015 Dec 01;1(2):e17. [doi: 10.2196/mededu.4886]

42. Applebaum P, Gutheil T. Clinical Handbook of Psychiatry and the Law, Fourth Ed. In: Confidentiality and Privilege. Philadelphia, PA: Lippincott Wilkins \& Williams; 2007:1-34.

Edited by G Eysenbach; submitted 30.04.20; peer-reviewed by MA Bahrami, C Jacob, A Louren, $R$ Zowalla; comments to author 12.06.20; revised version received 06.07.20; accepted 23.07.20; published 01.09.20

Please cite as:

Ahmed W, Jagsi R, Gutheil TG, Katz MS

Public Disclosure on Social Media of Identifiable Patient Information by Health Professionals: Content Analysis of Twitter Data J Med Internet Res 2020;22(9):e19746

URL: https://www.jmir.org/2020/9/e19746

doi: $10.2196 / 19746$

PMID: $\underline{32870160}$

(C)asim Ahmed, Reshma Jagsi, Thomas G Gutheil, Matthew S Katz. Originally published in the Journal of Medical Internet Research (http://www.jmir.org), 01.09.2020. This is an open-access article distributed under the terms of the Creative Commons Attribution License (https://creativecommons.org/licenses/by/4.0/), which permits unrestricted use, distribution, and reproduction in any medium, provided the original work, first published in the Journal of Medical Internet Research, is properly cited. The complete bibliographic information, a link to the original publication on http://www.jmir.org/, as well as this copyright and license information must be included. 